\title{
An ACA accelerated isogeometric boundary element analysis of potential problems with non-uniform boundary conditions
}

\author{
Lucas Silveira Campos ${ }^{\mathrm{a}}$, Éder Lima de Albuquerque ${ }^{\mathrm{a}}$, Luiz Carlos Wrobel $^{\mathrm{a}}$ \\ ${ }^{a}$ Universidade Federal do Esprito Santo, Departamento de Engenharia Mecânica, Esprito Santo, Brasil \\ ${ }^{b}$ Universidade de Brasília, Faculdade de Tecnologia, Departamento de Engenharia Mecânica, Distrito Federal, Brasil \\ ${ }^{c}$ Brunel University London, Institute of Materials and Manufacturing, Uxbridge UB8 3PH, UK
}

\begin{abstract}
This paper presents an Adaptive Cross Approximation (ACA) accelerated Isogeometric Boundary Element Method (IGBEM) using Non-Uniform Rational B-Splines (NURBS) as shape and interpolation functions. Provided that NURBS are used in CAD programs to describe geometry, mesh generation in the IGBEM is no longer necessary. For large and complex models the traditional BEM quickly becomes very time and memory consuming. In order to overcome this problem, the use of ACA is considered in this paper. As the NURBS control points are typically located outside the boundary, non-uniform boundary conditions cannot be applied at control points. So, a transformation matrix is used to allow the application of boundary conditions at control points without losing accuracy and, with a special approach, preserving the time and memory advantages of hierarchical matrices provided by the ACA. Two and tree dimensional numerical examples are presented in order to assess the accuracy and feasibility of the method.

Keywords: Boundary Element Method, Isogeometric analysis, ACA
\end{abstract}

\section{Introduction}

The predominant technology used by CAD packages in the representation of complex geometries is the rational nonuniform B-spline (NURBS). This allows to accurately reproduce entities that would only be approximated by polynomial functions, including circular conic sections. There is a vast literature dealing with different aspects of NURBS (eg: [⿴囗⿰丿㇄口, [2]), and decades of research have resulted in several efficient algorithms for rapid assessment and refinement. The main concept presented in [3] is to use NURBS not only to represent the geometry, but also as a discretization tool in the analysis, assigning the denomination of isogeometric analysis to this family of methods. Later, a book was published [4], dedicated exclusively to isogeometric analysis using the finite element method (FEM). This type of analysis

Email addresses: lucas.s.campos@ufes.br (Lucas Silveira Campos), eder@unb.br (Éder Lima de Albuquerque), luiz.wrobel@brunel.ac.uk (Luiz Carlos Wrobel) 
has already been applied in several areas, including solids mechanics, fluid mechanics and mechanical contact. The idea of using CAD technologies in the FEM has already been discussed since [5] where B-splines are used as shape functions in the FEM. In the BEM, a formulation using B-splines was presented initially in [6] and [7] but still without concern for integration into CAD. This integration was later observed in [8] and [9].

Although more developed in the FEM context, the isogeometric concept is only really isogeometric in the BEM. In CAD models only the surfaces are defined, and the interior of the solids are not explicitly modelled. However, in FEM, since the domain definition is required for the analysis, an additional stage where CAD representations have to be converted to solid models is required. Meanwhile, in BEM, only the boundary representation is needed, which is completely defined by CAD.

In addition, the higher-order continuity of NURBS basis functions reduces the sparsity of the matrices in the FEM, greatly increasing the computational cost of the method [10]. This effect is not observed in the BEM, since the matrices generated are already full. Another disadvantage of the isogeometric FEM is that the Gauss quadrature is no longer optimal since the integrand is no longer a simple polynomial. This behaviour is already observed even in the standard BEM since the fundamental solution, which is part of the integrand, is not a polynomial.

The idea that the BEM is superior to domain methods since they reduce the size of the problem by discretizing only its surface is somewhat simplistic. Matrices generated by the method are full, non-symmetric and have a high assembly cost. Several techniques have been investigated in order to overcome these difficulties: wavelets [II], block-based solvers [12, 13, 14] agglutination processes, and iterative techniques [15, 16]. A popular technique is the multipole method [17, 18, 19, 20, 21, 22, 23, 24, 25]. The fast multipole method, in spite of making the BEM more efficient for large scale problems, requires that a series expansion of the fundamental solution must first be known. All the terms of the series, for a given precision, must be calculated beforehand and then integrated, which significantly alters the standard BEM integration process. Another very popular technique is based on adaptive cross-approximation (ACA) [26, 27, 28, 29]. This technique efficiently reduces the assembly time and storage cost of the BEM matrices without altering the standard BEM integration process, provided it is an algebraic approach.

The ACA technique approximates the BEM dense matrices by hierarchical matrices, which allows a representation with a much lower amount of data. This representation is based on a tree structure that describes a block partitioning of the matrix. Each block contains a low rank approximation or the exact matrix itself. References [30, 31, 32] define the arithmetic of hierarchical matrices including operations such as addition, multiplication, inversion and LU factorization using the advantages obtained by low rank approximations.

To solve a system of linear equations with many degrees of freedom, iterative methods [33] should be applied in order to achieve superior performance. Any of the classical methods, such as Jacobi, Gauss-Seidel, minimum residue (MINRES), conjugate gradient, bi-stabilized conjugate gradient (BICGSTAB) or generalized minimum residue (GMRES), 
has as its main cost the matrix-vector multiplication that is much more efficient when using hierarchical matrices. A BEM code that uses ACA, hierarchical matrices and iterative techniques can greatly accelerate the time required by the simulations.

The use of ACA to accelerate isogeometric BEM can be found in the literature (see, for example, [34]). However, this previous work presents only the analysis of numerical problems where boundary conditions are uniform. On the other hand, they do not present any special treatment to apply non-uniform boundary conditions. As it will be shown in this paper, provided that boundary conditions in the isogeometric formulation are applied at control points, it is necessary to use a technique to move boundary conditions from boundary points to control points. Otherwise, only uniform boundary condition problems could be analysed satisfactorily.

This work presents an isogeometric formulation along with fast boundary elements, using the ACA as a fast method and NURBS as isogeometric basis functions. No work has been found in the literature where an isogeometric formulation of the BEM is used in conjunction with fast methods and applied to problems with non-uniform boundary conditions. Therefore, the proposed formulation has the advantages inherent to both ACA and IGBEM: being an accelerated method that skips the mesh generation step and has exact geometric representation.

\section{NURBS}

A NURBS curve is defined by its order, a set of weighted control points, and a knot vector. NURBS curves and surfaces are generalizations of both B-splines and Bezier curves and surfaces. The primary difference is the weighting of the control points due to the fact that the NURBS curves are rational (non-rational B-splines are a special case of rational B-splines).

The shape of the curve is determined by control points. Each point of the curve is computed by taking a weighted sum of a number of control points multiplied by basis functions. For a curve of degree $d$, the basis function of any control point is only nonzero in $d+1$ intervals of the parameter space. Within those intervals, the basis functions change according to a rational polynomial function. At boundaries of intervals, basis functions go smoothly to zero, the smoothness being determined by the degree of the polynomial.

The knot vector is a sequence of parameter values that determines where and how control points affect the NURBS curve. The number of knots is equal to the number of control points plus the order of the curve. The knot vector divides the parametric space in intervals, referred to as knot spans. Each time the parameter value enters a new knot span, a new control point becomes active, while an old control point is discarded.

Consecutive knots can have the same value. This defines a knot span of zero length, which implies that two control points are activated at the same time (and consequently two control points become deactivated). This alters the con- 
tinuity of the curve allowing the creation of corners in an otherwise smooth NURBS curve. A number of coinciding knots is known as a knot with a certain multiplicity. The multiplicity of a knot is limited to the degree of the curve, since a higher multiplicity would split the curve into disjoint parts and would leave control points unused.

The B-spline basis functions used in the construction of NURBS curves is denoted as $N_{i, k}(u)$, in which $i$ corresponds to the $i$-th control point, and $k$ corresponds to the degree of the basis function. The function is computed as

$$
N_{i, k}(u)=\frac{u-t_{i}}{t_{i}+k-t_{i}} N_{i, k-1}(u)+\frac{t_{i+k+1}-u}{t_{i+k+1}-t_{i+1}} N_{i+1, k-1}(u)
$$

and

$$
N_{i, 0}(u)=\left\{\begin{array}{l}
1, \text { if } t_{i}<=u<t_{i+1} \\
0, \text { otherwise }
\end{array}\right.
$$

The definition of these basis functions is clearly recursive.

Once these functions are defined, one can calculate the piecewise rational polynomial function that is the NURBS basis function:

$$
C(u)=\sum_{i=0}^{n} \frac{w_{i} P_{i} N_{i, k}(u)}{w_{i} N_{i, k}(u)}
$$

A NURBS surface representation is a straightforward extension of a NURBS curve. By carrying out a tensor product between two sets of basis functions one can obtain the surface $S(u, v)$ as:

$$
S(u, v)=\frac{\sum_{i=0}^{n} \sum_{j=0}^{m} N_{i, p}(u) N_{j, q}(v) w_{i, j} P_{i, j}}{\sum_{i=0}^{n} \sum_{j=0}^{m} N_{i, p}(u) N_{j, q}(v) w_{i, j}} .
$$

It is worthwhile to use a rational basis definition, such as:

$$
R_{i, j}(u, v)=\frac{N_{i, p}(u) N_{j, q}(v) w_{i, j}}{\sum_{k=0}^{n} \sum_{l=0}^{m} N_{k, p}(u) N_{l, q}(v) w_{k, l}}
$$

With this definition, the surface equation can be simplified to:

$$
S(u, v)=\sum_{i=0}^{n} \sum_{j=0}^{m} R_{i, j}(u, v) P_{i, j}
$$


Equation (G) is the one that will be used in the isogeometric analysis, and relates values at the control points to values in the surface.

\section{The boundary element method}

The isogeometric boundary element method differs from the conventional BEM on three main points:

- The basis functions for the geometry and field variables,

- The position of the collocation points,

- The application of the boundary conditions.

A direct collocation form of the BEM consists in constructing a system of equations by taking a collocation point for every unknown and integrating along the boundary. For potential problems, the integral equation that relates the potential and its derivative is given as:

$$
c u\left(x^{\prime}\right)=\int_{\Gamma} \frac{\partial u}{\partial n} u^{*} d \Gamma-\int_{\Gamma} u \frac{\partial u^{*}}{\partial n} d \Gamma
$$

where $c$ is a jump term that arises from the limiting process of the integral equation and is dependent on the geometry at the source point $x^{\prime}, u$ and $\frac{\partial u}{\partial n}$ are the potential and its derivative in the normal to the boundary direction, respectively, while $u^{*}$ and $\frac{\partial u^{*}}{\partial n}$ refers to fundamental solutions.

In order to make equation $(\mathbb{Z})$ suitable for numerical implementation, the continuous fields $u$ and $\frac{\partial u}{\partial n}$ have to be represented in a discrete manner. In the conventional BEM, the idea is to split the boundary into elements using a Lagrange interpolation while in the isogeometric method the geometry is exactly obtained from the CAD model and the basis functions from equations (Bi) and (6) are used to approximate the variables. This leads to:

$$
c u\left(x^{\prime}\right)=\int_{\Gamma} u^{*} \sum_{i=1}^{n+1}\left(\frac{\partial u_{i}^{c}}{\partial n} R_{i, k}(t)\right) d \Gamma-\int_{\Gamma} \sum_{i=1}^{n+1}\left(u_{i}^{c} R_{i, k}(t)\right) \frac{\partial u^{*}}{\partial n} d \Gamma .
$$

The boundary integrals in the conventional BEM can be easily divided into elements whereas in isogeometric formulations the definition of elements is not obvious. An easy way to define elements is by separating the knot spans in domains of influence of each control point, resulting in:

$$
c u\left(x^{\prime}\right)=\sum_{i=1}^{n+1}\left(\frac{\partial u_{i}^{c}}{\partial n} \int_{t_{i}}^{t_{i+k}} u^{*} R_{i, k}(t) \frac{d \Gamma}{d t} d t\right)-\sum_{i=1}^{n+1}\left(u_{i}^{c} \int_{t_{i}}^{t_{i+k}} R_{i, k}(t) \frac{\partial u^{*}}{\partial n} \frac{d \Gamma}{d t} d t\right) .
$$


where, finally, one can do a transformation to a standard domain in order to easily integrate numerically using GaussLegendre quadrature:

$$
\begin{aligned}
\sum_{i=1}^{n+1}\left(u_{i}^{c}\left(\int_{-1}^{1} R_{i, k}(t) \frac{\partial u^{*}}{\partial n}\left(x, x^{\prime}\right) \frac{d \Gamma}{d t} \frac{d t}{d \xi} d \xi+c R_{i, k}\left(x^{\prime}\right)\right)\right)= & \\
& \sum_{i=1}^{n+1}\left(\frac{\partial u_{i}^{c}}{\partial n} \int_{-1}^{1} u^{*}\left(x, x^{\prime}\right) R_{i, k}(t) \frac{d \Gamma}{d t} \frac{d t}{d \xi} d \xi\right) .
\end{aligned}
$$

The Jacobian of the transformation comprises of two terms: one that maps from the physical coordinate space, $\frac{d \Gamma}{d t}$, and one that maps from the parameter space of the curve to a local coordinate $\frac{d t}{d \xi}$.

The same straightforward procedure can be applied to potential and elasticity in both two and three dimensions. For three dimensions, the resulting equation is given as:

$$
\begin{aligned}
\sum_{i=1}^{n+1}\left(u_{i}^{c}\left(\int_{-1}^{1} \int_{-1}^{1} R_{i, k}(t) \frac{\partial u^{*}}{\partial n} J \frac{d t}{d \xi} \frac{d v}{d \eta} d \xi d \eta+c R_{i, k}\left(x^{\prime}\right)\right)\right) & = \\
& \sum_{i=1}^{n+1}\left(\frac{\partial u_{i}^{c}}{\partial n} \int_{-1}^{1} \int_{-1}^{1} u^{*} R_{i, k}(t) J \frac{d t}{d \xi} \frac{d v}{d \eta} d \xi d \eta\right)
\end{aligned}
$$

where the surface integrals are transformed into double integrals in a standard domain and $J$ is the Jacobian of the transformation from global coordinates $x, y, z$ to local coordinates $t, v$.

\subsection{Integration}

The evaluation of boundary integrals is key to any BEM implementation. However, in isogeometric BEM, special care is needed since the strongly singular integrals cannot be obtained indirectly through the constant temperature or rigid body motion hypothesis. The values at the collocation points are not interpolated as in the conventional BEM. They are approximated, resulting in multiple singular integrals for each equation. The method of choice for dealing with strongly singular integrals is the singularity subtraction technique presented by [35] for two-dimensional problems and by [36] for three-dimensional problems. Weakly singular integrals are calculated using the Telles [37] transformation in two dimensions, while in three dimensions the singular area integral is dealt with by transforming it into polar coordinates.

The choice of collocation points used in this work makes it simple to obtain the jump term $c$ since their position will always be on a smooth boundary, resulting in a value of $c$ always equal to $1 / 2$. 


\subsection{Collocation points}

Control points and the knots used to define the NURBS are not required to be unique, as mentioned earlier; they can have a multiplicity up to the degree of the curve. Control points are not located at the boundary but on a circumscribed polygon. These characteristics make these entities not the best choices for collocation points in the BEM. The domain point over which a control point has maximal influence is called a Greville abscissa [38] and is defined as an average of $d$ knots :

$$
\gamma_{i}=\frac{1}{d}\left(t_{i+1}+\ldots+t_{i+d}\right) .
$$

where $d$ is the degree of the curve and $\gamma_{i}$ are the Greville coordinates. These points are unique, located at the boundary and are equal in number to the control points. When the curve is smooth, these are the ideal candidates to be used as collocation points, and are used in such manner in [8] and [39]. However, when corners are present, specially for 3D problems, it becomes troublesome to calculate the diagonal terms since the collocation points are at corners.

To circumvent this issue, collocation points are, in this work, distributed according to Gaussian quadrature points:

$$
\gamma_{i}=\frac{\left(t_{i+1}-t_{i+2}\right)(\xi+1)}{2}+t_{i+1}
$$

where $\xi$ is the coordinate of the Gauss points. If all knots are unique, the number of knot spans, with length different from zero, is equal to the number of control points and only one Gauss point is used. Therefore, the collocation points will be at the center of each knot span. When the knot has a multiplicity larger than one, the number of Gauss points is increased, maintaining an equal number of collocation points and control points.

Considering that the Gauss points are never at the limits of the integration interval, collocation points will also never be at a corner of the curve.

This procedure resembles the use of discontinuous elements but no change in the control points is made and, consequently, there is no loss of continuity in the approximation.

\section{Adaptive cross approximation}

The ACA acceleration process starts by dividing the geometric domain as a tree data structure. This division creates, in each tree node, a cluster of collocation points or control points that are close to each other. When the distance between a pair of collocation and control points is large enough, the block defined from those entries is considered admissible 
and is represented by a low rank approximation. The remaining blocks are generated in the usual BEM manner and are stored as full matrices. A detailed presentation of ACA can be found in [40] and [41]].

The savings in memory and time are due to the low rank approximations. This format needs less entries than a full matrix and is much faster when multiplying a vector. Most iterative methods for solving linear systems are based on matrix-vector multiplication and if that is optimised, the whole method will become more efficient.

The procedure involved in calculating the low rank approximations in ACA has to evaluate exactly a column and a row at a time. For constant elements, this can be done easily as each column is related to a specific element and therefore also a specific node. When dealing with isogeometric BEM, there is no proper definition of element and each control point has a unique, but intersecting, interval in which its basis function is non-zero.

That interval can be understood as the domain of influence $\Omega_{i}$ of each control point $i$ and is defined as:

$$
\Omega_{i}=\left(t_{i+1}, t_{i+d}\right)
$$

To calculate a single column, there is only the need to integrate the domain of influence of the corresponding control point. By working in such manner, the basis function and the geometric properties of Gauss points are only calculated once for each column. It is important reminding that the evaluation of NURBS basis functions is much more expensive than regular polynomial basis functions. When calculating a row, it is necessary to integrate over all domains of influence of the corresponding control points. By definition, when one seeks a low rank approximation, field and source points are relatively far from each other, therefore one can expect that fundamental solutions will change smoothly leading to the possibility of using a very low number of Gauss points, half the order of the curve plus one for example, and still obtaining a satisfactory approximation.

\section{Boundary conditions}

As control points are typically outside the boundary, boundary conditions cannot be applied directly. In order to overcome this issue, a transformation matrix $\mathbf{E}$ for B-splines was proposed in [6]. This matrix uses the basis functions to relate values at control points with values at collocation points:

$$
\begin{array}{r}
\mathbf{u}=\mathbf{E u}_{c} \\
\frac{\partial \mathbf{u}}{\partial \mathbf{n}}=\mathbf{E} \frac{\partial \mathbf{u}_{\mathbf{c}}}{\partial \mathbf{n}}
\end{array}
$$


where $\mathbf{u}$ is a vector containing values at the collocation points and $\mathbf{u}_{c}$ is the vector containing values at control points. An equivalent matrix can be obtained for NURBS using the equilibrium equation derived in [6]:

$$
\mathbf{H u}_{c}=\mathbf{G} \frac{\partial \mathbf{u}_{\mathbf{c}}}{\partial \mathbf{n}}
$$

which is rewritten as:

$$
\mathbf{H E}^{-1} \mathbf{u}=\mathbf{G E}^{-1} \frac{\partial \mathbf{u}}{\partial \mathbf{n}}
$$

This procedure becomes an issue when one tries to use ACA. The inverse of the transformation matrix is not sparse and the method would become unable to be accelerated by ACA, as the hierarchical matrices $\mathbf{H}$ and $\mathbf{G}$ would turn into full matrices after being multiplied by $\mathbf{E}^{-1}$.

What is proposed is that we add a knot with maximum multiplicity for every change in boundary condition type. By doing so, intervals with different boundary conditions can be separated and values at control points can be found by solving a simple linear system for each interval:

$$
\begin{gathered}
\mathbf{u}_{c}=\left[\mathbf{E}_{0}^{-1} \mathbf{u}_{0} \cdots \mathbf{E}_{i}^{-1} \mathbf{u}_{i} \cdots \mathbf{E}_{n}^{-1} \mathbf{u}_{n}\right] \\
\frac{\partial \mathbf{u}_{\mathbf{c}}}{\partial \mathbf{n}}=\left[\mathbf{E}_{0}^{-1} \frac{\partial \mathbf{u}_{\mathbf{0}}}{\partial \mathbf{n}} \cdots \mathbf{E}_{i}^{-1} \frac{\partial \mathbf{u}_{\mathbf{i}}}{\partial \mathbf{n}} \cdots \mathbf{E}_{n}^{-1} \frac{\partial \mathbf{u}_{\mathbf{n}}}{\partial \mathbf{n}}\right]
\end{gathered}
$$

This procedure does not change the geometry but points where the boundary condition changes become only $C_{0}$ continuous. For most cases, that should not be an issue.

\section{RESULTS}

In this session, in order to assess the potential isogeometric formulation, two two-dimensional and four three-dimensional problems are analysed. Results are compared with constant elements and the analytical solution. For the 3D problems, results for the conventional BEM are obtained with the software provided by [25], that uses constant triangular elements and the fast multipole method. In order to assess the accuracy at single points, the relative error $e_{r e l}=\frac{y_{t}}{y_{a}}$ is considered. The normalized root mean square error is used as an error measure in some instances. This estimator is 
given by:

$$
e_{r m s}=\frac{\sqrt{\frac{\sum_{t=1}^{n}\left(y_{t}-y_{a}\right)^{2}}{n}}}{y_{\max }-y_{\min }} .
$$

where $y_{t}$ is the numerically obtained value at point $t, y_{a}$ is the analytical value, $n$ is the number of points, $y_{\max }$ and $y_{\min }$ are the maximum and minimum values of variable $y_{a}$.

\subsection{Heat transfer in a hollow cylinder}

The first problem is the heat transfer in a hollow cylinder that will be modelled in two dimensions as shown in figure Ш. This problem has an analytical solution which will be used to assess the results. The temperature is known at the internal boundary $S_{i}$ and the flux is known at the external boundary $S_{e}$. The analytical solution for the temperature is given by:

$$
T(r)=T_{i}+q_{e} r_{e} \log \left(\frac{r}{r_{i}}\right)
$$

and for the flux:

$$
q(r)=q_{e} \frac{r_{e}}{r}
$$

where $T_{i}$ e $q_{e}$ are the temperature and the flux at the internal and external boundaries, respectively.

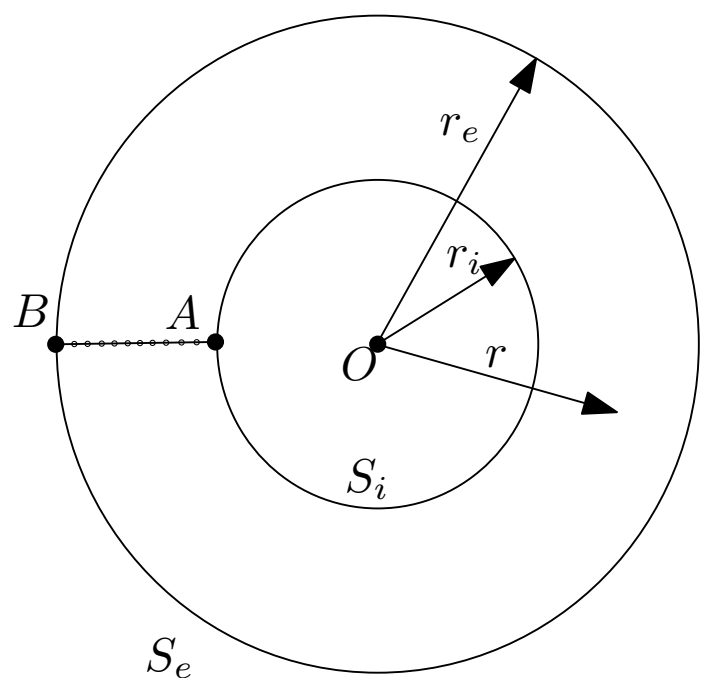

Figure 1: Potential problem in an annular region.

For this problem, the following values are considered: $r_{i}=1, r_{e}=2, T_{i}=100, q_{e}=200$ and $k=1$. This results in the analytical solution $T_{e}=377.25$ and $q_{i}=-400$. The external and internal boundaries are discretized with the same number of elements. In figures $\square$ and 及, relative errors for the temperature at point $B$ and flux at point $A$ are shown for constant and isogeometric elements. 


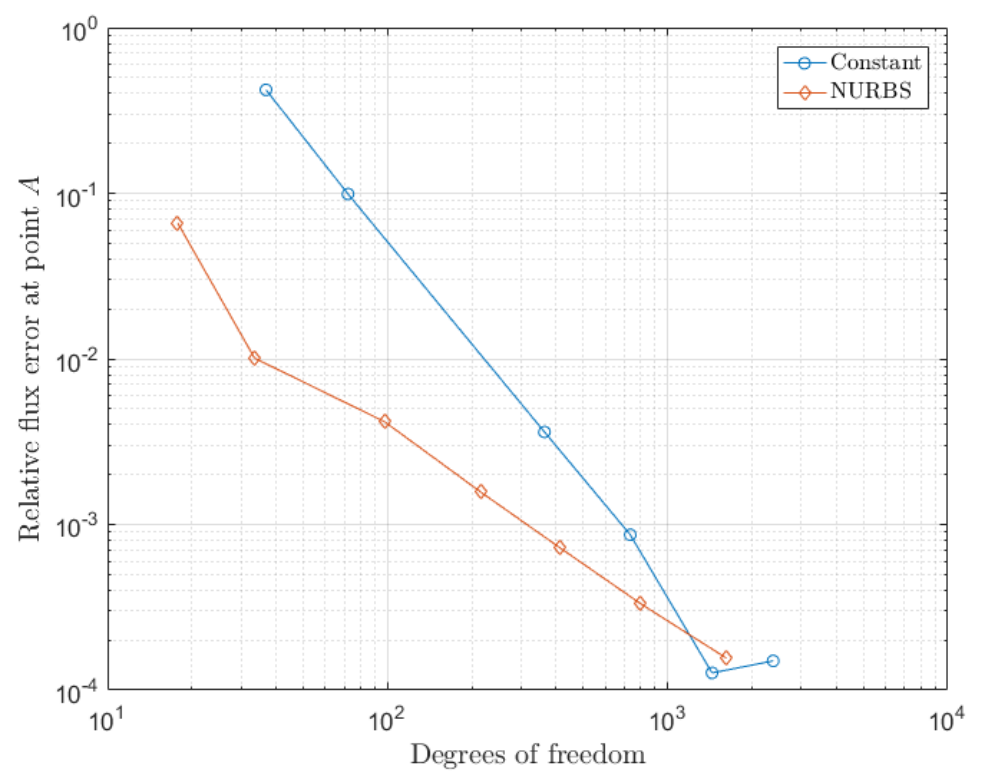

Figure 2: Flux errors at $A$.

Both the conventional method and the isogeometric method converge to the exact solution. The isogeometric discretization has a better response with few elements while constant elements have a faster convergence. As all variables are constant, the biggest difference between the methods is the geometric representation. Even the poorest discretization represents the geometry exactly for the isogeometric method.

RMS errors are used to show the approximation at internal points. The temperature is calculated at 10 uniformly distributed points along the segment $A B$. The RMS error and the maximum error are shown in figure 4 .

The error obtained by the isogemetric method is smaller than the constant even for a number of nodes where the error at the boundary for the conventional method is smaller. This shows that the exact representation of the boundary in the isogeometric method has stronger influence on internal points than on boundary points. Reasons for this behaviour are out of the scope of this work.

\subsection{Rectangular plate}

The problem analysed is a rectangular plate as shown in figure 5 .

Boundary conditions are:

$$
\begin{aligned}
& q=-\frac{1}{2 \sqrt{r}}\left(\cos \frac{\theta}{2} \cos \theta+\sin \frac{\theta}{2} \sin \theta\right) \text { in } B C, \\
& q=-\frac{1}{2 \sqrt{r}}\left(\cos \frac{\theta}{2} \cos \theta-\sin \frac{\theta}{2} \sin \theta\right) \text { in } C D,
\end{aligned}
$$




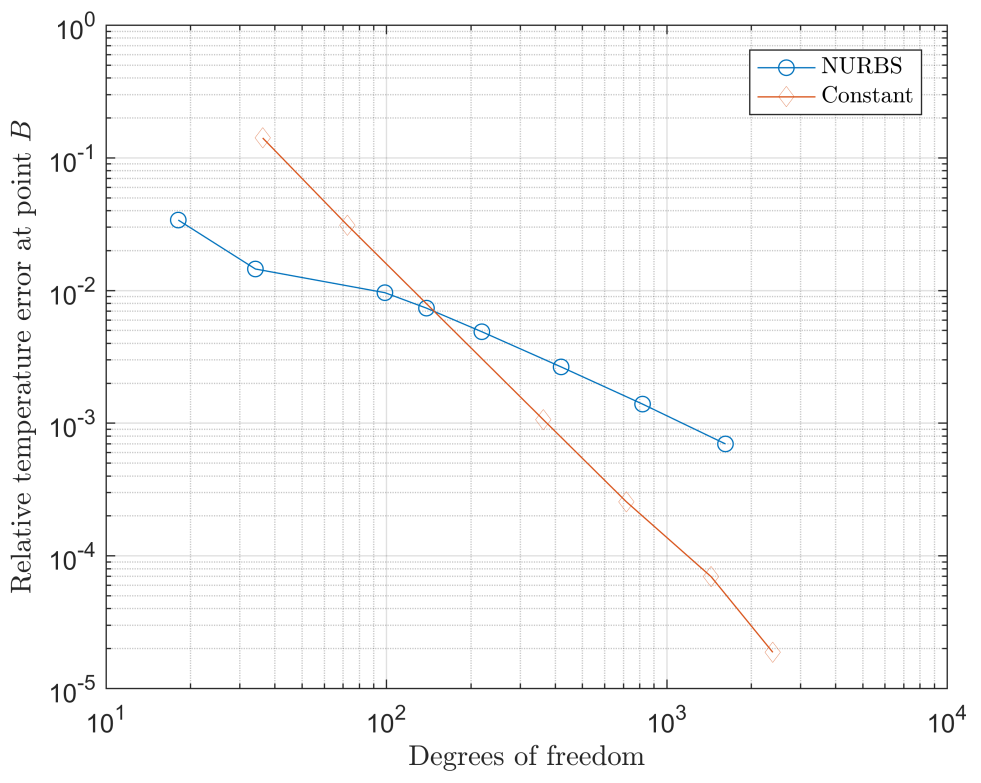

Figure 3: Temperature errors at $B$.

$$
q=\frac{1}{2 \sqrt{r}}\left(\cos \frac{\theta}{2} \cos \theta+\sin \frac{\theta}{2} \sin \theta\right) \text { in } D A,
$$

$$
T=0 \text { in } A O
$$

and

$$
q=0 \text { in } O B .
$$

This problem has an analytical solution given by:

$$
\begin{gathered}
u=\sqrt{r} \cos \frac{\theta}{2} \\
q_{x}=\frac{\cos \frac{\theta}{2}}{2 \sqrt{r}}
\end{gathered}
$$

and

$$
q_{y}=\frac{\sin \frac{\theta}{2}}{2 \sqrt{r}}
$$

Figures 6 and $\square$ show the relative error of the temperature at point $E$ for isogeometric elements of various orders and constant elements. In figure 6, the proposed method for imposing the boundary conditions is utilized, while in figure $\square$ no special care is taken. There is a clear difference: results obtained with the proposed method are superior to the 


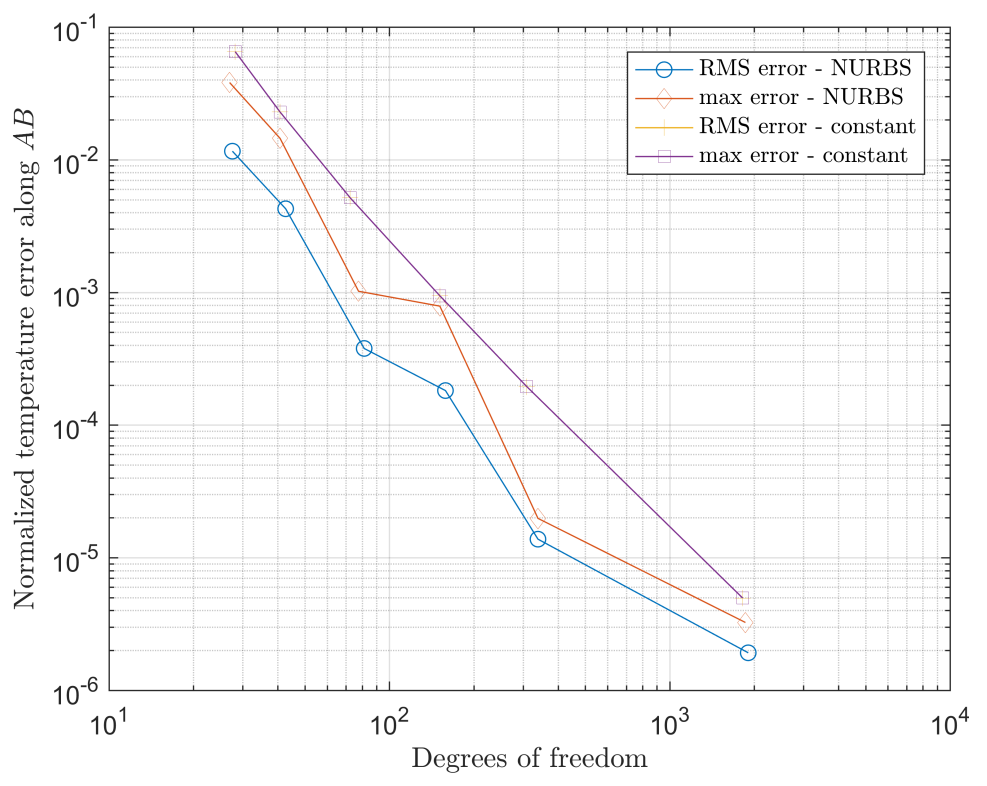

Figure 4: Temperature errors along $A B$.

alternative. This is a good problem to show the efficiency of the method as the imposed boundary conditions are not uniform. They are, in fact, quite complex, as there is a singularity at point $O$.

Results in figure 6 show an improvement as the order increases. However, even for the isogeometric element with the lowest order, 2, there is an improvement when compared to the conventional method.

\subsection{Sphere}

The example shown in [42] will be analysed here. The problem consists in a spherical structure of radius equal to 1 and centre at $(0.5,0.5,0.5)$ as shown in figure $\mathbb{\nabla}$. The temperature prescribed at the boundary is:

$$
u=x^{2}-y^{2}+2 x+z
$$

The analytical solution for the flux is given by $-k \mathbf{n} \nabla u$ where:

$$
\nabla u=\left[\begin{array}{lll}
2 x+2 & -2 y & 1
\end{array}\right]
$$

In table [, the normalized RMS errors are shown for both isogeometric elements of order 3 and constant triangular elements. In this comparison, it becomes clear that the performance of isogeometric elements is superior to standard constant boundary elements. However, the NURBS representation of the sphere generates two poles that concentrate 


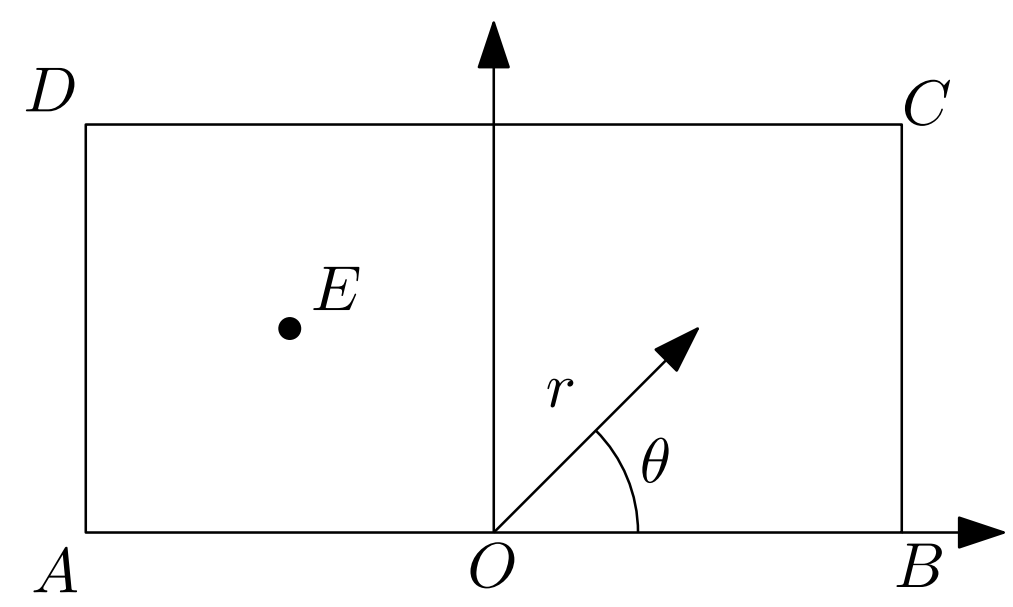

Figure 5: Rectangular plate.

Table 1: Normalized RMS error for the sphere.

\begin{tabular}{|c|c|c|c|}
\hline \multicolumn{2}{|c|}{ Constant elements } & \multicolumn{2}{c|}{ Order 3 isogeometric elements } \\
\hline Nodes & Normalized RMS Errors & Nodes & Normalized RMS Errors \\
\hline 320 & 0.017847 & 45 & 0.0110208 \\
1280 & 0.011417 & 91 & 0.00231124 \\
5120 & 0.010104 & 153 & 0.00151761 \\
20480 & 0.0099437 & & \\
\hline
\end{tabular}

the control points, as seen in figure 8. This concentration requires special attention in dealing with the singularities. The treatment of the singular integrals suffer with element distortion [43] requiring over 500 integrations points, when near the poles, in order to obtain an error smaller than $10^{-6}$. In future works, special techniques to reduce the amount of integration points in these kind of problems should be addressed.

\subsection{Torus}

In this example, presented by [42], a torus of external radius $R=2$ and internal radius $r=1$ is analysed with the temperature at the boundary equal to:

$$
u=\frac{x^{2}}{2}+\frac{y^{2}}{2}-z^{2}+5 x+5 y+5 z .
$$

The analytical solution for the flux is given by $-k \mathbf{n} \nabla u$, where:

$$
\nabla u=\left[\begin{array}{lll}
x+5 & y+5 & -2 z+5
\end{array}\right]
$$

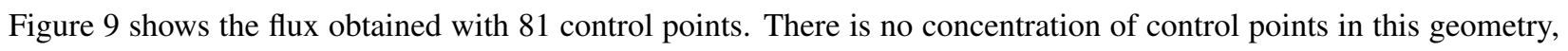
that leads to a less troublesome singularity treatment when compared to the sphere (a maximum of 120 integration 


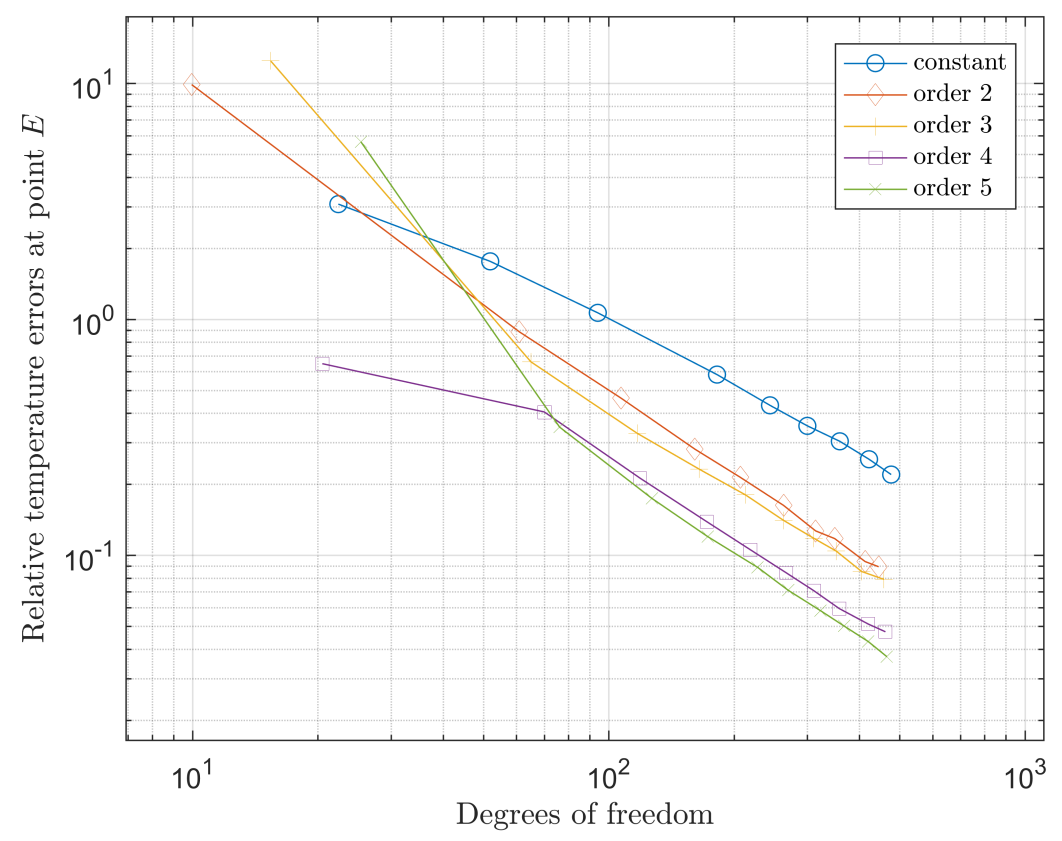

Figure 6: Errors at point $E$ for the proposed method.

Table 2: Normalized error for the torus

\begin{tabular}{|c|c|c|c|}
\hline \multicolumn{3}{|c|}{ Constant elements } & \multicolumn{2}{c|}{ Order 3 isogeometric elements } \\
\hline Nodes & Normalized RMS error & Nodes & Normalized RMS error \\
\hline 1792 & 0.0086708 & 81 & 0.0176615 \\
28224 & 0.0098135 & 169 & 0.0027632 \\
& & 441 & 0.000568215 \\
\hline
\end{tabular}

points is used).

In table ఇ, where the RMS errors for the collocation points are shown, a clear advantage of the isogeometric method can be observed when compared to the constant element. In [42] a comparison is made with quadratic elements where, even with the use of 1600 elements, the error is still higher than $10^{-3}$.

\subsection{Square plate with holes}

In order to test the efficiency of ACA in the execution time of the method, a square plate with a growing number of uniformly distributed holes is analysed. In figure 10, one can observe the case for 16 holes. The size of the holes is modified in such a way that the ratio between the area of the holes and the area of the plate remains constant and equal to $12.47 \%$.

The execution time as the number of holes increases, from 1 until 121, is shown in figure $\square$. The change of slope of the curves shows the evident improvement in speed: the standard method has a slope close to $n^{2}$ while the one using 


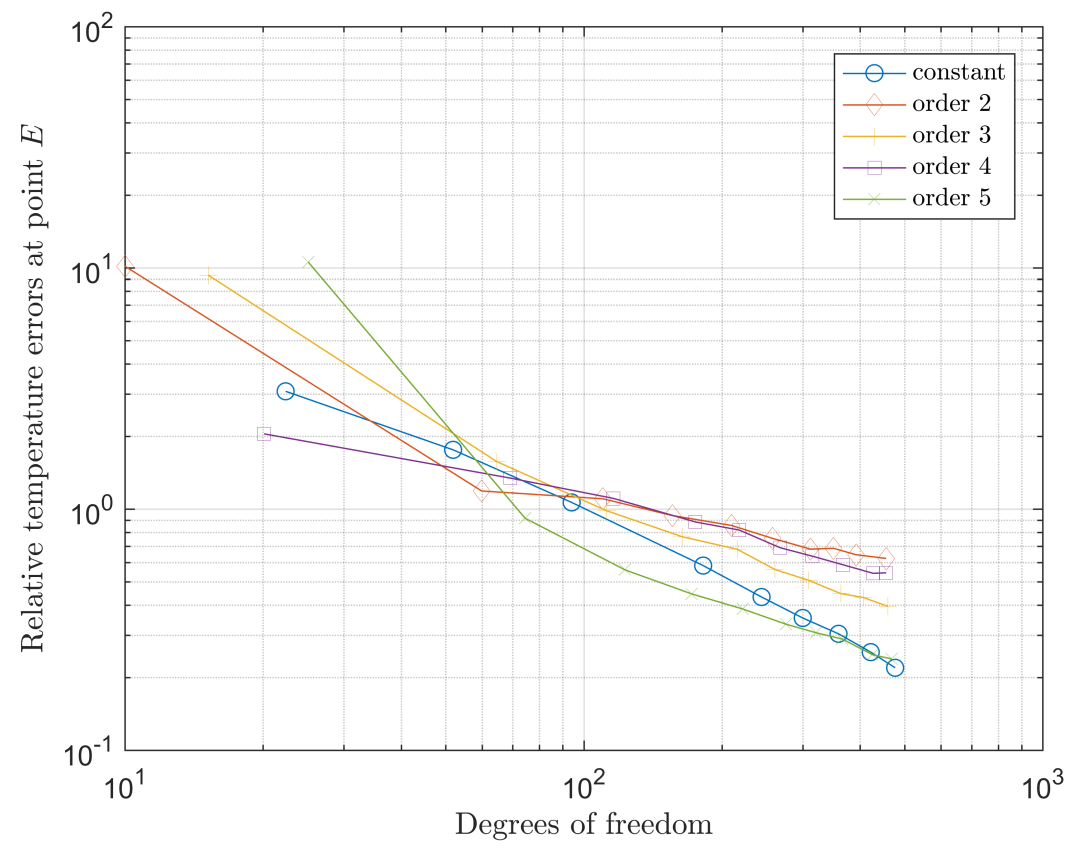

Figure 7: Errors at point $E$ without the proposed method.

ACA is close to $n \log n$. Even though the accelerated method is slower for small problems, the different in slope make it faster for larger problems.

\section{CONCLUSION}

This work presented a proper way to couple NURBS and ACA, taking special care with boundary conditions, resulting in a method that is very efficient for large and complex CAD models with generic and non-uniform boundary conditions. The usage of ACA provides memory saving and running time reduction when compared to the standard BEM. As it uses the CAD parameters directly, there is no need to create a mesh in the isogeometric formulation. This drastically reduces the engineering work involved in the numerical analysis.

The position of the collocation points makes the formulation easier to implement, specially for three dimensional problems, since there is no collocation point at corners. As the curve is always smooth, there is no need for a special treatment of the diagonal terms. However, quasi-singularities are present, since points are near the corners. When there is a concentration of the control points, as in the sphere, the integrals become more troublesome.

The method for imposing the boundary conditions is shown to be accurate while maintaining the beneficial properties of hierarchical matrices. The order of complexity of the method reduces from $n^{2}$ to $n \log n$ by taking advantage of 


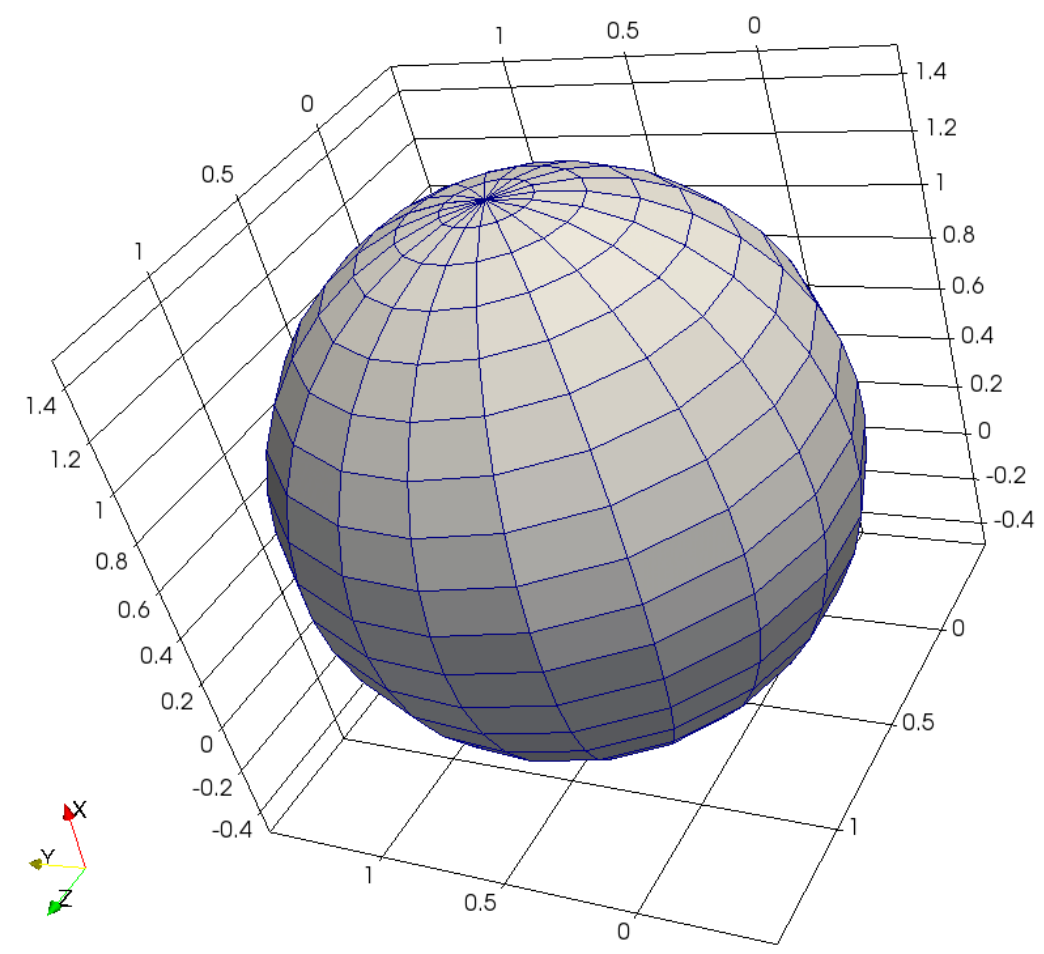

Figure 8: Sphere represented with NURBS.

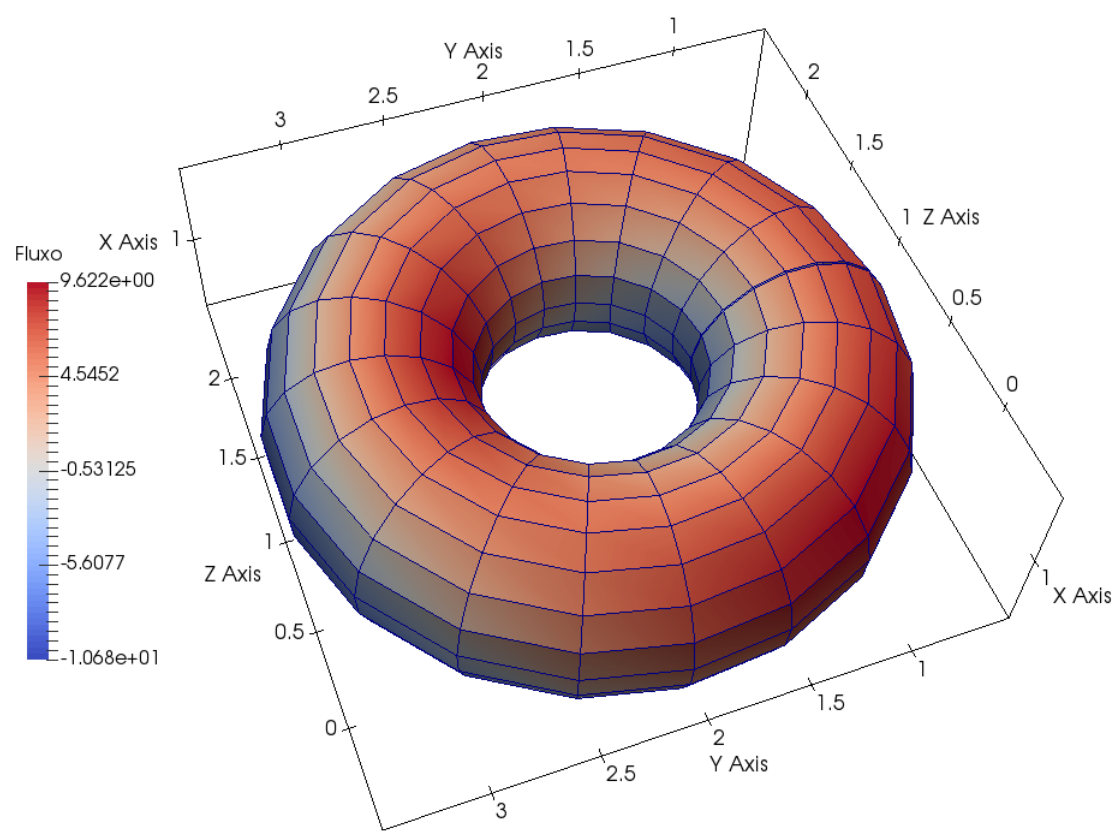

Figure 9: Torus represented by NURBS. 


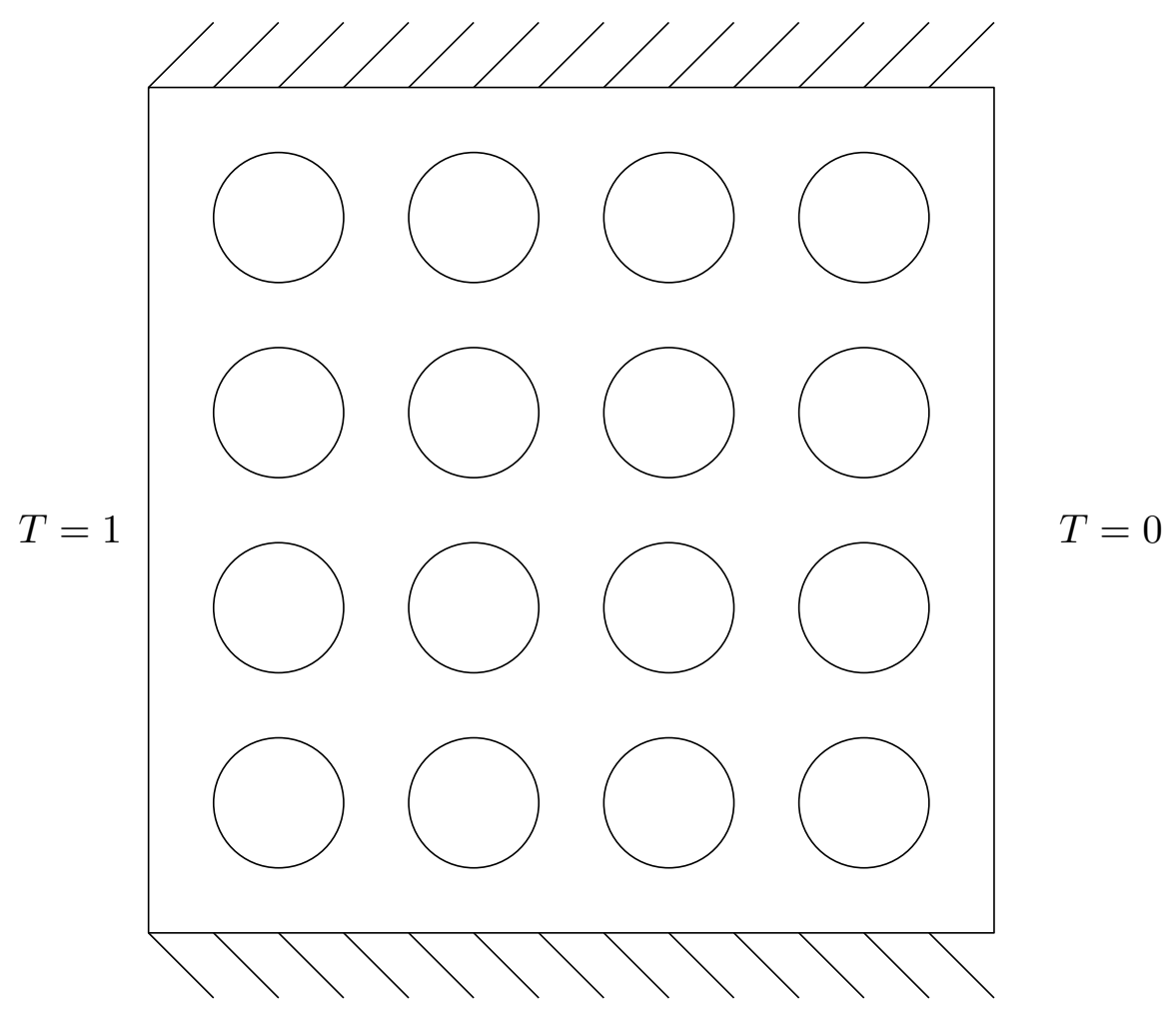

Figure 10: Plate with holes.

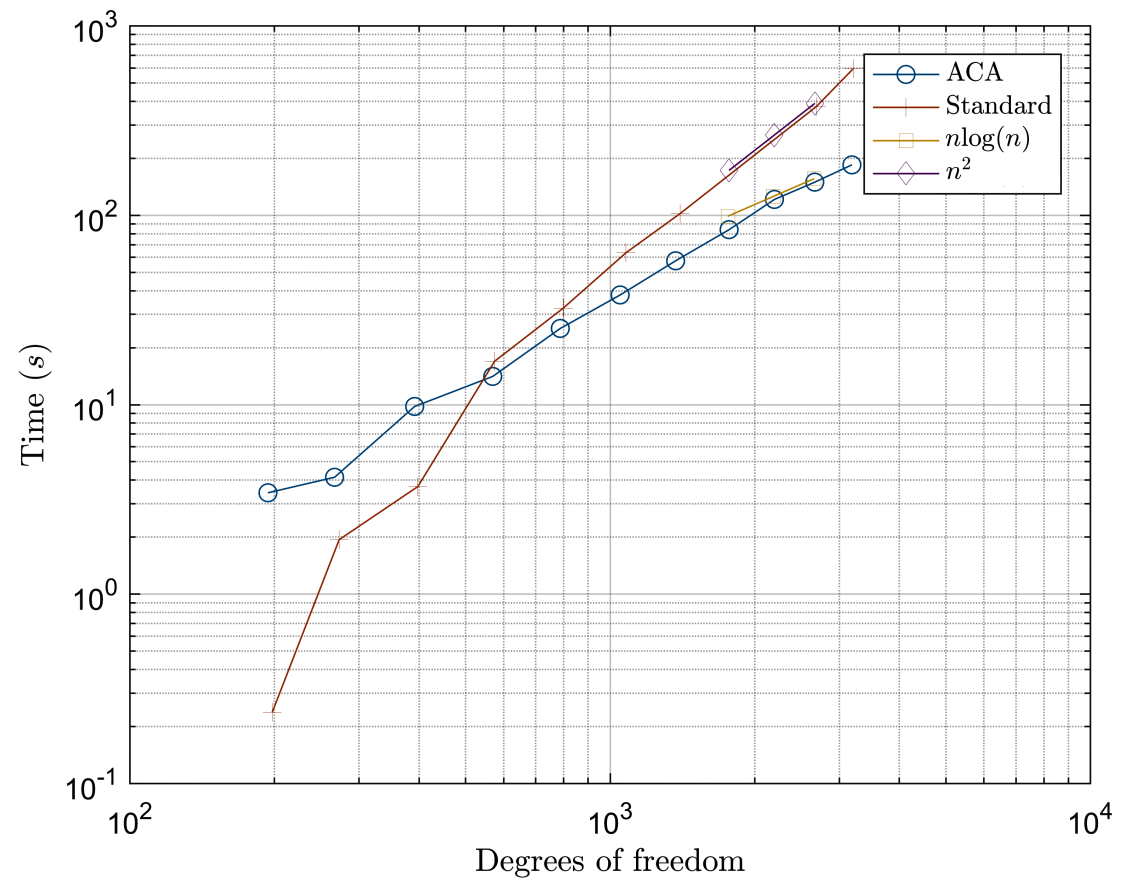

Figure 11: Execution time for various holes. 
the ACA. The proposed method is not only faster and more memory efficient but also more accurate than the standard BEM.

\section{References}

[1] L. Peigl, W. Tiller, The NURBS book, Berlin, Springer-Verlag, 1996.

[2] D. F. Rogers, An introduction to NURBS: with historical perspective, Elsevier, 2000.

[3] T. J. Hughes, J. A. Cottrell, Y. Bazilevs, Isogeometric analysis: CAD, finite elements, NURBS, exact geometry and mesh refinement, Computer Methods in Applied Mechanics and Engineering 194 (39) (2005) 4135-4195.

[4] J. A. Cottrell, T. J. R. Hughes, Y. Bazilevs, Isogeometric analysis - toward integration of CAD and FEA, Wiley, 2009.

[5] P. Kagan, A. Fischer, Integrated mechanically based CAE system using B-Spline finite elements, Computer-Aided Design 32 (8) (2000) 539-552.

[6] J. J. S. P. Cabral, L. C. Wrobel, C. A. Brebbia, A BEM formulation using B-splines: I-uniform blending functions, Engineering Analysis with Boundary Elements 7 (3) (1990) 136-144.

[7] J. J. S. P. Cabral, L. C. Wrobel, C. A. Brebbia, A BEM formulation using B-splines: II-multiple knots and non-uniform blending functions, Engineering Analysis with Boundary Elements 8 (1) (1991) 51-55.

[8] R. N. Simpson, S. P. A. Bordas, J. Trevelyan, T. Rabczuk, A two-dimensional isogeometric boundary element method for elastostatic analysis, Computer Methods in Applied Mechanics and Engineering 209 (2012) 87-100.

[9] R. N. Simpson, S. P. A. Bordas, H. Lian, J. Trevelyan, An isogeometric boundary element method for elastostatic analysis: 2D implementation aspects, Computers \& Structures 118 (2013) 2-12.

[10] N. Collier, D. Pardo, L. Dalcin, M. Paszynski, V. M. Calo, The cost of continuity: a study of the performance of isogeometric finite elements using direct solvers, Computer Methods in Applied Mechanics and Engineering 213 (2012) 353-361.

[11] H. F. Bucher, L. C. Wrobel, W. Mansur, C. Magluta, A novel approach to applying fast wavelet transforms in the boundary element method, Electronic Journal of Boundary Elements 2 (2002) 187-195.

[12] R. Rigby, M. Aliabadi, Out-of-core solver for large, multi-zone boundary element matrices, International Journal for Numerical Methods in Engineering 38 (9) (1995) 1507-1533.

[13] J. Crotty, A block equation solver for large unsymmetric matrices arising in the boundary integral equation method, International Journal for Numerical Methods in Engineering 18 (7) (1982) 997-1017. 
[14] J. Kane, B. Kumar, S. S. Kashava, An arbitrary condensing, noncondensing solution strategy for large scale, multi-zone boundary element analysis, Computer Methods in Applied Mechanics and Engineering 79 (2) (1990) 219-244.

[15] W. Mansur, F. Araujo, J. Malaghini, Solution of BEM systems of equations via iterative techniques, International Journal for Numerical Methods in Engineering 33 (9) (1992) 1823-1841.

[16] L. Barra, A. Coutinho, W. Mansur, J. Telles, Iterative solution of BEM equations by GMRES algorithm, Computers \& Structures 44 (6) (1992) 1249-1253.

[17] S. Aoki, K. Amaya, M. Urago, A. Nakayama, Fast multipole boundary element analysis of corrosion problems, CMES: Computer Modeling in Engineering \& Sciences 6 (2) (2004) 123-131.

[18] J. Carrier, L. Greengard, V. Rokhlin, A fast adaptive multipole algorithm for particle simulations, SIAM Journal on Scientific and Statistical Computing 9 (4) (1988) 669-686.

[19] H. Cheng, L. Greengard, V. Rokhlin, A fast adaptive multipole algorithm in three dimensions, Journal of Computational Physics 155 (2) (1999) 468-498.

[20] W. Chew, J. Song, T. Cui, S. Velamparambil, M. Hastriter, B. Hu, Review of large scale computing in electromagnetics with fast integral equation solvers, Computer Modeling in Engineering and Sciences 5 (4) (2004) $361-372$.

[21] L. Greengard, V. Rokhlin, A fast algorithm for particle simulations, Journal of Computational Physics 73 (2) (1987) 325-348.

[22] X. He, K.-M. Lim, S.-P. Lim, Fast BEM solvers for 3D Poisson-type equations, CMES: Computer Modeling in Engineering \& Sciences 35 (1) (2008) 21-48.

[23] V. Rokhlin, Rapid solution of integral equations of classical potential theory, Journal of Computational Physics 60 (2) (1985) 187-207.

[24] H. Wang, Z. Yao, A new fast multipole boundary element method for large scale analysis of mechanical properties in 3D particle-reinforced composites, Computer Modeling in Engineering and Sciences 7 (1) (2005) 85-95.

[25] Y. Liu, Fast multipole boundary element method: theory and applications in engineering, Cambridge University Press, 2009.

[26] W. Hackbusch, Hierarchical Matrices: Algorithms and Analysis, Springer, 2016.

[27] M. Bebendorf, R. Grzhibovskis, Accelerating Galerkin BEM for linear elasticity using adaptive cross approximation, Mathematical Methods in the Applied Sciences 29 (2006) 1721-1747. 
[28] M. Bebendorf, S. Rjasanow, Adaptive low-rank approximation of collocation matrices, Computing 70 (2003) $1-24$.

[29] M. Bebendorf, Approximation of boundary element matrices, Numerische Mathematik 86 (2000) 565-589.

[30] W. Hackbusch, A sparse matrix arithmetic based on h-matrices. Part I, International Journal for Numerical Methods in Engineering 62 (1999) 89-108.

[31] W. Hackbusch, A sparse matrix arithmetic based on h-matrices. Part II, International Journal for Numerical Methods in Engineering 64 (2000) 21-47.

[32] L. Grasedyck, W. Hackbusch, Construction and arithmetics of H-matrices, Computing 70 (4) (2003) 295-334.

[33] A. Greenbaum, Iterative methods for solving linear systems, Vol. 17, SIAM, 1997.

[34] B. Marussig, J. Zechner, G. Beer, T.-P. Fries, Fast isogeometric boundary element method based on independent field approximation, Computer Methods in Applied Mechanics and Engineering 284 (2015) 458-488.

[35] M. Guiggiani, G. Krishnasamy, T. Rudolphi, F. Rizzo, A general algorithm for the numerical solution of hypersingular boundary integral equations, Journal of Applied Mechanics 59 (3) (1992) 604-614.

[36] C. J. B. Ubessi, Método dos elementos de contorno para elasticidade linear 3D com avaliação direta das integrais singulares, Msc Dissertation, UFRGS, Brazil (in portuguese).

[37] J. C. F. Telles, A self-adaptive co-ordinate transformation for efficient numerical evaluation of general boundary element integrals, International Journal for Numerical Methods in Engineering 24 (5) (1987) 959-973.

[38] G. E. Farin, Curves and Surfaces for Computer-Aided Geometric Design: A Practical Code, Academic Press, Inc., 1996.

[39] M. Scott, R. N. Simpson, J. Evans, S. Lipton, S. P. A. Bordas, T. Hughes, T. Sederberg, Isogeometric boundary element analysis using unstructured T-splines, Computer Methods in Applied Mechanics and Engineering 254 (2013) 197-221.

[40] M. Bebendorf, Hierarchical matrices: a means to efficiently solve elliptic boundary value problems, Vol. 63, Springer, 2008.

[41] S. Kurz, O. Rain, S. Rjasanow, Fast Boundary Element Methods in Computational Electromagnetism, in: Boundary Element Analysis, Springer, 2007, pp. 249-279.

[42] Y. Zhang, Y. Gong, X. Gao, Calculation of 2D nearly singular integrals over high-order geometry elements using the sinh transformation, Engineering Analysis with Boundary Elements 60 (2015) 144-153.

[43] K. Hayami, H. Matsumoto, Improvement of quadrature for nearly singular integrals in 3D-BEM, WIT Transactions on Modelling and Simulation 7 (1994) 10. 